Published in

Ikeda, A., Xu, H., Fuji, N., Zhu, S., \& Yamada, Y. (2019). Questionable research practices following pre-registration. Japanese Psychological Review, 62(3), 281-295.

\title{
Questionable research practices following pre-registration
}

\author{
Ayumi Ikeda ${ }^{1 *}$, Haoqin $\mathrm{Xu}^{1}$, Naoto Fuji ${ }^{1}$, Siqi Zhu ${ }^{1}$, \\ and Yuki Yamada ${ }^{1,2 * *}$ \\ ${ }^{1}$ Graduate School of Human-Environment Studies, Kyushu University, Japan \\ ${ }^{2}$ Faculty of Arts and Science, Kyushu University, Japan
}

*Corresponding author 1: Ayumi Ikeda

Address: Graduate School of Human-Environment Studies, Kyushu University,

744 Motooka, Nishi-ku, Fukuoka 819-0395, Japan

E-mail: hnthnt4444@gmail.com

TEL \& FAX: +81-92-802-5837

**Corresponding author 2: Yuki Yamada

Address: Faculty of Arts and Science, Kyushu University,

744 Motooka, Nishi-ku, Fukuoka 819-0395, Japan

E-mail: yamadayuk@gmail.com

Twitter:@momentumyy

TEL \& FAX: +81-92-802-5837 


\begin{abstract}
The credibility of psychological findings can be undermined by a history of questionable research practices (QRPs) of researchers. One remedy for this problem is the use of the pre-registration of studies in which a research protocol is registered before the experiment starts. However, the current style of pre-registration can be negatively affected by other QRPs. The purpose of this paper was to demonstrate the ability to engage in QRPs even after a study has been pre-registered. In the demonstration study, we used a total of eight QRPs to obtain statistically meaningful results that supported an ad hoc hypothesis. Major system updates such as pre-registration, peer review, and evaluation are required to address these harmful practices. We hope that the present demonstration study gives momentum to further discussions on next-generation research practices.
\end{abstract}

Keywords: open science, pre-registration, transparency, questionable research practices, embodied cognition 
Scientific theories must be based on reliable evidence, and the replicability of research results is essential for demonstrating reliability. However, in recent years, it has been reported that the success rate of direct replication is low in many research fields, such as medicine (Ioannidis, 2005), economics (Camerer, Dreber, Forsell, Ho, Huber, Johannesson et al., 2016), and cognitive neuroscience (Szücs \& Ioannidis, 2017). Further, this issue has been identified in criminology (Lösel, 2017), computational sciences (Bernard, 2017), and experimental philosophy (Colombo, Duev, Nuijten, \& Sprenger, 2018; Cova, Strickland, Abatista, Allard, Andow, Attie et al., 2018). Psychology is at the center of this topic, as psychological research results also generally have low rates of replicability. In 2015, a multi-lab study in the journal $\underline{\text { Science }}$ reported that only $36 \%$ of direct replications of psychological studies that were published in prominent journals (i.e., Psychological Science, Journal of Personality and Social Psychology, and Journal of Experimental Psychology: Learning, Memory, and Cognition) were successful (Open Science Collaboration, 2015, see also Klein, Vianello, Hasselman, Adams, Adams, Alper et al., 2018). Furthermore, a well-known social psychologist performed a series of experimental results claiming that humans could foresee the future (Bem, 2011), but multiple replications of these experiments failed (e.g., Galak, LeBoeuf, Nelson, \& Simmons, 2012; Ritchie, Wiseman, \& French, 2012). Moreover, it was revealed that another social psychologist, Diederik Stapel, garnered accolades and academic achievements by fabricating data for ten years. Thus, the replicability problem in psychology has become known to both scientists and the general public worldwide and directly challenges the ability to regard psychology as a science.

Nevertheless, academic journals continue to have little room for replication studies. The current severity of the replicability problem has become more apparent, which suggests a possibility that journals do not highly regard replications supporting the validity of evidence. Additionally, the “publication bias” (Mahoney, 1977; Sterling, Rosenbaum, \& Weinkam, 1995), which makes it challenging to publish null results in journals continues to exist. It is such a serious problem that researchers are obliged to seek significant results even using various questionable research practices 
(QRPs). One of these is $\underline{p}$-hacking or the practice of trying to make $\underline{p}$ values low. A previous study (Simmons, Nelson, \& Simonsohn, 2011) demonstrated this QRP in an experiment in which they examined the hypothesis that listening to a Beatles' song would lead to listeners becoming significantly younger. The significant results were produced by modifying data handling $(\underline{p}<.05)$, which highlights the fact that even absurd hypotheses can be supported by $\underline{p}$-hacking. $\underline{P}$-hacking includes such practices as adding new participants and including them in the statistical analyses until significant results are obtained. Likewise, cherry picking (Fraser, Parker, Nakagawa, Barnett, \& Fidler, 2018) occurs both intentionally and inadvertently. Researchers tend to select evidence that can support their claims and will then hide or ignore the remaining inconsistent evidence. Such concealed and dishonest practices have been repeatedly reported (Greenwald, Pratkanis, Leippe, \& Baumgardner, 1986; Strube, 2006). Also, researchers sometimes construct a working hypothesis after the results of experiments are shown to make a "good" story, which has been referred to as HARKing ("hypothesizing after the results are known;" Kerr, 1998). HARKing is a relatively accepted practice in psychology, and Bem (2004) suggested this as a good way to write a paper. These QRPs increase the probability of researchers making Type I errors.

Unfortunately, some researchers are inadequately aware of this problem and are insufficiently wary of the use of dubious research practices. A survey of 5964 researchers in the field of psychological science reported that more than 30\% had used QRPs at least once (John, Loewenstein, \& Prelec, 2012), which indicates that the use of QRPs has become widespread in psychology.

The pre-registration system is recommended by many psychologists as well as journals in other fields to prevent QRPs, as it functions as a platform that is used before the start of a study. Researchers can register a detailed plan of their study design and upload data continuously throughout the experiment on these pre-registration websites, such as Open Science Framework (OSF: https://osf.io/) or AsPredicted (https://aspredicted.org/). This process is designed to achieve the transparency of the whole study and prevent the occurrence of QRPs (e.g., Jonas \& Cesario, 2016; 
Mellor \& Nosek, 2018; Nosek, Spies, \& Motyl, 2012; Nosek et al., 2018; van’t Veer \& Giner-Sorolla, 2016). Recently, based on peer-reviewed pre-registration, some journals provide a de facto guarantee to publish registered reports regardless of the presence of significant results. More importantly, the pre-registration process seems to be able to distinguish prediction and postdiction accurately and clearly (Nosek et al., 2018), which protects against HARKing. Thus, this system could undoubtedly be a milestone in academic fields and greatly improve the authenticity and effectiveness of scientific and empirical research.

However, pre-registration does have some inherent weaknesses, as it cannot prevent some specific QRPs (Yamada, 2018). For example, researchers can repeat the registered experiments until obtaining a satisfactory result ("infinite re-experimenting," "reset marathon," or "rerolling") and make a reasonable hypothesis that is "inferred" based on observations before pre-registration (PARKing; Pre-registering After the Results are Known, see also Boccia et al., 2016; Szücs, \& Ioannidis, 2017). Since PARKing intentionally breaks the temporal order between pre-registration and obtaining results, it reintroduces the possibility of all traditional QRPs. Moreover, researchers can deliberately choose to only report things that they want from many privately registered protocols. Multiple similar experimental protocols can be registered on different pre-registration websites, and only the significant studies that can be reported and published are completed while the rest are abandoned (overissuing). This QRP is especially problematic and easy to do in systems where third parties cannot search for registered protocols, such as AsPredicted.

These issues are not parallel situations; rather, they are complexly related and mutually interacting. These problems arise from only one simple cause: every researcher wants to be an "incredible researcher." Research achievement is evaluated based on how many sensational discoveries are made, how many positive research results are obtained, and how many published articles have appeared in top-tier journals. However, often researchers have limited time, and research does not always go well, which can lead to dishonesty. 
Although pre-registration itself has no intrinsic ethical nature, it is problematic to use preregistration to either implicitly or explicitly conflate meaning that "pre-registered = good paper," without considering the possibility that, as a system, it can be cracked through deliberate efforts (Yamada, 2018). Even in studies using pre-registration, research misconduct such as fabrication, falsification, and plagiarism (FFP), as well as QRPs may appear. Researchers need to be aware of both the pros and cons of the pre-registration system and use it appropriately.

For the reasons above, the problems with the pre-registration system have been considered in the present paper. Yamada (2018) mentioned the cracks in the pre-registration system, but those concerns were presented in an opinion paper. To promote the awareness of the importance of properly using a pre-registration system throughout the scientific community, there was a need to demonstrate the ability to engage in QRPs for pre-registration. Thus, we devised a demonstration of an experimental study that used many QRPs, as was done by Simmons et al. (2011) and Fujishima and Higuchi (2016). However, we did not engage in deliberate research misconduct such as FFP, as we wanted to illustrate the ease of implementing these bad behaviors and the difficulty in recognizing them, rather than being concerned about our actual experimental findings (using FFP). In other words, we aimed to shed light

on the danger of these QRPs that have historically been viewed as being in the "gray zone." The next section presents the demonstration study; its contents are hoax, and it includes many QRPs. Subsequently, we will explain what types of QRPs were involved in the demonstration study and what that means.

\title{
Demonstration Study
}

\section{Right preference of right-handers in a negative state}

\begin{abstract}
The purpose of this study was to clarify how emotional state and sensorimotor information interact in preferential decision making. The experimental protocol was pre-registered on AsPredicted. In the
\end{abstract}


study, the emotional state of right-handed participants was measured while they selected a hit card three separate times from two facedown cards, using Positive and Negative Affect Schedule. Participants with a strong negative emotional state were significantly more likely to choose the right card for the third choice than were participants with a weak negative emotional state, suggesting that people with a highly negative state prefer to use their dominant hand.

\section{Introduction}

Stressful situations change people's behavior and cognition. Suicide, violence, sexual impulses, and drug use may become more aggressive or conservative in stressful situations (Ayduk, Downey, Testa, Yen, \& Shoda, 1999; Erwin, Heimberg, Schneier, \& Liebowitz, 2003; Kashdan, Collins, \& Elhai, 2006; Shaffer, Gould, Fisher, Trautman, Moreau, Kleinman, \& Flory, 1996). Kashdan et al. (2006) found that anxious people were more likely to engage in violent or dangerous sexual behavior to satisfy their strong desires, such as a sexual need, compared to those who were not anxious. Conversely, anxious people are more inclined to consider the consequences of their actions before they act when they have low-intensity desires, to control their dangerous behaviors effectively (Kashdan et al., 2006).

Why do people tend to behave impulsively when they have a strong desire? It is possible that when anxious people are eager to achieve a certain goal, they become defensive and eager to pursue results. In this situation, they consider less the consequences of the dangerous behavior, thereby reducing the pain and punishment caused by the negative self-evaluation of the behavior. However, when anxious people do not have a certain desire, they stop the behavior because of the possibility of negative results (Kashdan et al., 2006). In cognitive experiments related to anxiety, anxious people's attention was found to be attracted by anxiety stimuli; that is, anxious people respond more rapidly to anxiety target stimuli than they respond to non-anxiety stimuli (e.g., Eysenck, 1982; Eysenck, Derakshan, Santos, \& Calvo, 2007; Staal, 2004). Thus, people with high anxiety use a stimulus-driven attentional system more than those with low anxiety (e.g., Corbetta \& Shulman, 2002; Posner \& 
Petersen, 1990), so that their decisions are dominated by anxiety stimuli in a bottom-up manner. Meanwhile, people with low anxiety tend to use a goal-directed attentional system to make top-down judgments. When there are many bottom-up factors around highly anxious people, their decision making is easily influenced by such factors (Decks \& Pegg, 2001 ${ }^{1}$ ).

Preference behaviors, a kind of decision-making, are embodied. This is explained based on the body-specificity hypothesis (Casasanto, 2009). Casasanto (2009) observed that emotional valence is related to people's physical proficiency. Specifically, right-handed people tended to place positive emotional words on the right side of their body-centered coordinates in a localization task and preferred a right-side object in an evaluation task. Meanwhile, left-handed people tend to place negative emotional words on their left side and prefer a left-side object (Casasanto, 2009; Casasanto \& Henetz, 2012; Casasanto \& Jasmin, 2010). In a subsequent study, this horizontal preference was found to be reversible (Casasanto \& Chrysikou, 2011). Right-handed people performed a 12-minute fluency task (e.g., moving or placing dominoes) with ski gloves, which disrupted the fluent action with their right hand. As a result, their horizontal preference was reversed. Moreover, researchers found that spatial bias for preference not only appeared on the horizontal axis but also more prominently on the vertical axis. Right-handed people preferred to put positive words and objects to right and upper, and this bias was robust and immune to participants' cultural context (Marmolejo-Ramos, Elosua, Yamada, Hamm \& Noguchi, 2013).

The experimental protocol of the present study was pre-registered on AsPredicted. During the pre-registration process, we indicated that the aim was to test whether the embodied left-right preference modulates highly anxious people's decision-making during a card-choice task (Livisio, $1988^{1}$ ). Participants were presented with two cards in the display and asked to choose a hit card (Figure 1). The two cards had the same appearance and were placed on the left or right of the screen. The choice task was repeated three times. The first and second choices aimed to give negative feedback to participants, so the program displayed the results as if the participants' answers were always incorrect 
in these choices, regardless of their choice. We designed the first two times to give participants negative feedback to make them feel anxious. Under the influence of the first two "incorrect" choices, the third choice took the role as an anxious state inducer. Here, it was predicted that participants would choose both the cards equally frequently in the first choice and that the choices would differ depending on the participants' PANAS-Negative score (Positive and Negative Affect Schedule; Watson, Clark, \& Tellegen, 1988). This is a subscale of PANAS that measures respondents' negative emotional state. In other words, we predicted that although participants with a low score in PANAS-Negative would choose both the cards with equal frequency, participants with a high PANAS-Negative score would choose a card on the side of the dominant hand more frequently. The first two "incorrect" choice would influence people who tend to feel strongly anxious, and in the final choice, they would choose without consideration, but depending more on embodied signals.

\section{Method}

Pre-registration. The protocol of the present study was registered before data collection (https://aspredicted.org/sv9nq.pdf). This experiment was performed the same way as was indicated on the pre-registered protocol. Details are shown in the following sub-sections.

Statistical analyses. In the pre-registered protocol, we planned to conduct Fisher's exact tests with the number of participants who selected the right card in the two participant groups.

Participants. The study participants would be divided into the highest quartile (top 25\%) and lowest quartile (bottom 25\%) based on their PANAS-Negative score. A power analysis using G*Power 3.1 (Faul, Erdfelder, Buchner, \& Lang, 2009) for 2.5 odds ratio with the required significance level $\alpha=$ 0.05 and the required power level $1-\beta=0.95$ suggested that the required sample size (of right-handers) was 336. The maximum sample size, including potential satisficers in crowdsourcing research (e.g., Chandler, Mueller, \& Paolacci, 2014; Oppenheimer, Meyvis, \& Davidenko, 2009; Sasaki \& Yamada, in press) and left-handers, was set at 500 for the stopping rule. Thus, we recruited 463 people online 
through a crowdsourcing service (Yahoo! Crowdsourcing: http://crowdsourcing.yahoo.co.jp/) and paid 22 T-points $(1 \mathrm{~T}$-point $=1 \mathrm{JPY})$ for their participation $(275$ men, Mean age $=43.82$ years, range 16-74, SD =9.96). The Internet-based study had been approved by the ethics committee of Kyushu University (approval number: QU-07121981).

Measures. Participants' affective state was measured by the Japanese version of PANAS (Kawahito, Otsuka, Kaida, \& Nakata, 2011), which consists of the 10-item Positive Affect scale (Cronbach's $\alpha$ $=.86$ ) and the 10-item Negative Affect scale (Cronbach's $\alpha=.89$ ). The items in this scale (e.g., $\underline{\text { Nervous }}, \underline{\text { Scared, }}, \underline{\text { Distressed }}, \underline{\text { Afraid }})$ were rated on a six-point Likert scale from 1 ( $\underline{\text { not at all })}$ ) to 6 ( $\underline{\text { very }}$ $\underline{m u c h}$ ). As we were interested in the degree of negative emotional state of participants, and as the number of items is large, participants' attentional allocation on the task declines, so only the PANASNegative items were used in this study.

Procedure. Figure 1 represents the time course of the experiment. First, participants were instructed to answer a questionnaire about demographic information (age; gender: male, female, or other; handedness: dextral, sinistral, or ambidextral). Next, participants were introduced to a card choice game, in which participants were asked to choose a card that was considered to have the "Bingo" mark behind it from two cards with the exact same appearance. One card was located on the left side of the screen and the other located on the right side of the screen on participants' own terminal equipment. After their decision, regardless of their answer, a sentence "Unfortunately, it's not Bingo" was displayed. This game was presented three times successively and induced a negative impression that participants failed to find the right answer during all three chances. In addition, the statement was presented as "Unfortunately, it's not Bingo. The next time is the last chance." at the end of the second failure choice. After that, participants completed the PANAS-Negative to assess their affective state. Three attention check questions (ACQ) were inserted into the items to confirm whether participants responded seriously. First, participants were asked to indicate "How many months are in a year" on a 6-point Likert scale ranging from 10 months to 15 months; second, they were asked to indicate "What 


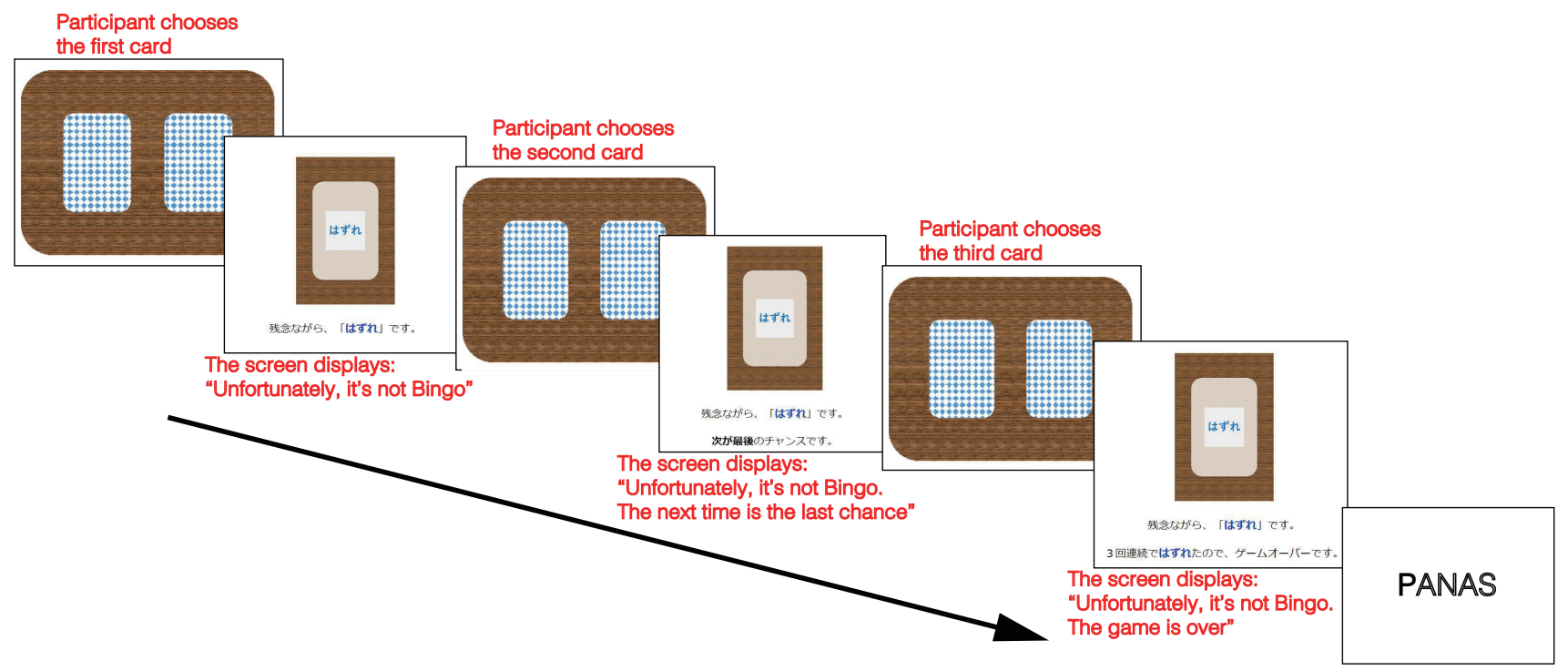

Figure 1. Schematic representation of the time course of the experiment

do koalas eat?" by selecting an answer from the following responses: bananas, Eucalyptus leaves, carrots, and potatoes; and third, they were asked to indicate "What color is the sky?" by choosing one option from the following responses: amber, aquamarine, black, and white.

\section{Results and discussion}

Analyses. All analyses were performed using R version 3.4.1 (R Core Team, 2017). Data from participants who mistook at least one of the three ACQs $(\underline{N}=47 ; 10.2 \%$ of the total $)$ and from lefthanders $(\underline{N}=24 ; 5.2 \%$ of the total) were not included in the analysis. Participants were divided into two groups. One group consisted of participants in the top $25 \%$ on the negative affect subscale (Cronbach's $\alpha=.93)$ and was named the strongly negative group $(\underline{N}=107)$, while the other consisted of participants who scored in the bottom $25 \%$ of the subscale and was named weakly negative group $(\underline{N}=100)$.

The data. All the present data, excluding the data from left-handers, are provided as a supplementary file: https://doi.org/10.6084/m9.figshare.7977035.v1). Fisher's exact test (using the fisher.exact() function of the exact $2 \times 2$ package) with the number of participants who chose the right card in the first and third choices as dependent variables was performed to examine the presence of a right preference in the card choice task. As a result, for the first choice, no significant difference was found in this test 
$(\underline{p}>.40)$. The odds ratio of the strongly and weakly negative groups was 1.27 (95\% CI [0.74, 2.20]). On the other hand, for the third choice, a significant difference was found $(\underline{p}<.02)$. The odds ratio of the strongly and weakly negative groups was 1.97 (95\% CI [1.13,3.43]) and indicated that participants in the strongly negative group were significantly more likely to choose the right card in the third choice than those in the weakly negative group (Figure 2). 

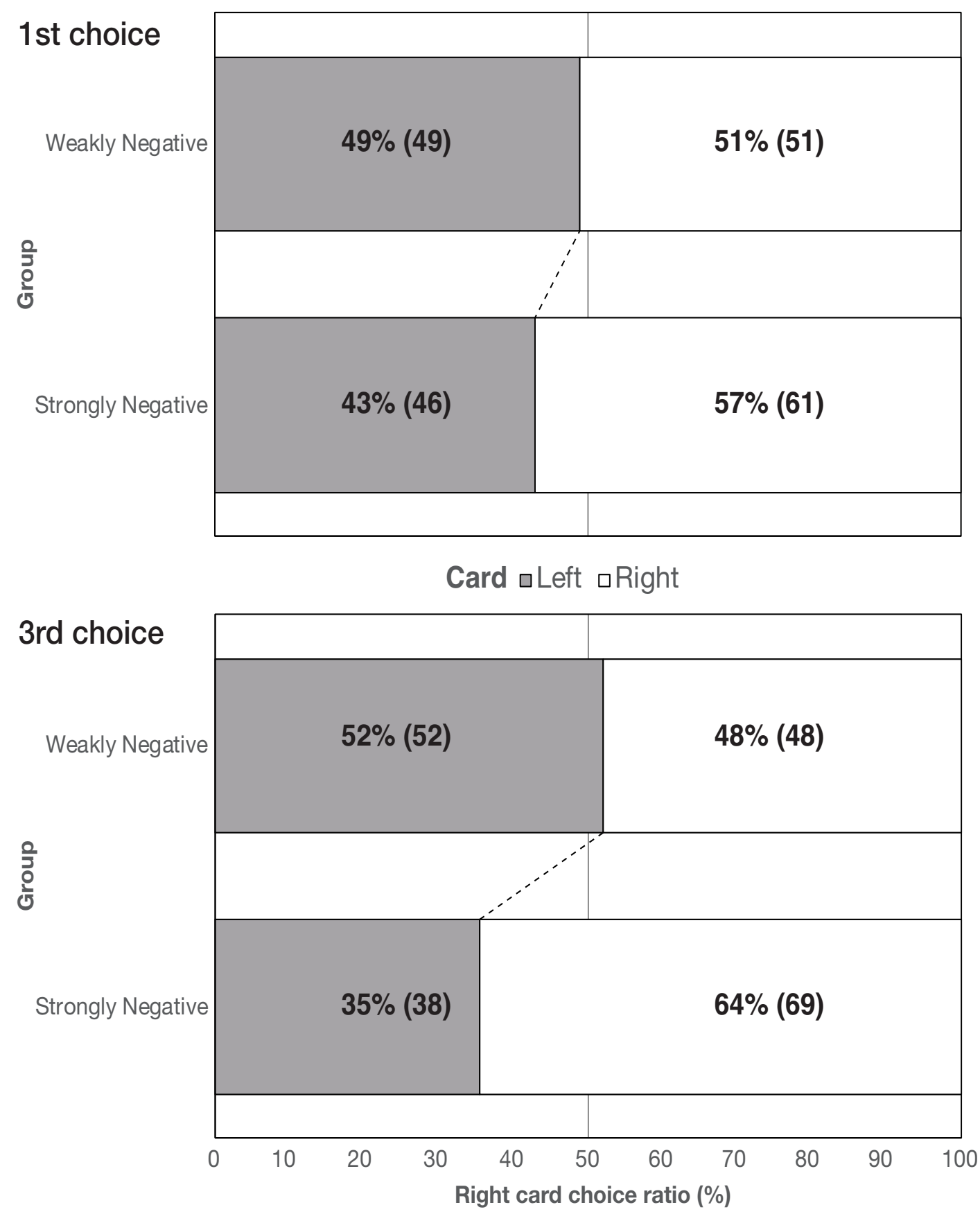

Figure 2. The choice ratio of the right cards in each group

Interpretation. The results were consistent with the prediction and supported our hypothesis; that is, right-handers tended to prefer the right side when they were in a negative state. In the third choice of the present card choice game, the affective state of participants in the strongly negative group should be relatively more negative than in the first choice because of the continuous failures and therefore resulting in the right preference. These results suggested that participants' choice behavior was based on the body-specificity hypothesis (i.e., right-handers like right; Casasanto, 2009). More interestingly, 
this effect was modulated by the current affective state. Namely, bodily and affective signals would be crossed before the signals are delivered to the decision-making process, maybe depending on the availability of cognitive resources.

Limitations. Some limitations remain. First, it is not clear whether the results of the present study will occur only in right-handers. This point is quite important for the explanation based on the bodyspecificity hypothesis: if left-handers also showed the same tendency, then it means that the present findings should not be related to the affective mapping on the body space. Unfortunately, in the present study, there are considerable difficulties involved in collecting data from numerous sinistral participants, but further investigation with such a sample is required. Second, the present experiment was conducted online, and hence, it is unclear what kind of screen was used by each participant while engaging in the task (Saramista, Nelli, Nous, \& Vinicoso, 2015 $)$. The position of the cards in the card task in the present study strongly depends on the screen size. Laboratory experiments may be necessary to fix the size of the screen.

\section{General Discussion}

The above sections introduced a very ordinary study in experimental psychology focused on the influence of an embodied emotion on the choice behavior. However, the results were false positives. We chose embodiment as a mock topic of research for two reasons. First, this topic is often doubted when considering the replicability of the results. For example, as a representative case, Williams and Bargh (2008) demonstrated that perceived temperatures affect the perceiver's interpersonal behavior or impression formation. However, their experiments have repeatedly failed during direct replication (Chabris, Heck, Mandart, Benjamin, \& Simons, 2018; Lynott et al., 2014). Note that this statement is entirely unrelated to individual cases cited in the demonstration study, such as Casasanto's several works (one of them is directly replicated in this special issue; Sasaki, Yonemitsu, \& Yamada, in press). Second, we selected a method that can show very simple results. In the method of the present 
demonstration study, it is easy to obtain significant results only by the coincidental presence of an unbalanced number of people. In addition, here, we used various kinds of QRPs. We did register the present study on the OSF, detailing the various QRPs we would use to produce apparently significant results (https://osf.io/jmd3s/). We even called the study "Meta-Prereg" during this process. This motivation of this paper was to demonstrate that new QRPs for manipulating the pre-registration system (Yamada, 2018) as well as traditional QRPs (e.g., Agnoli, Wicherts, Veldkamp, Albiero, \& Cubelli, 2017; Motyl, Demos, Carsel, Hanson, Melton, Mueller et al., 2017; Munafò, Nosek, Bishop, Button, Chambers, du Sert et al., 2017; Simmons et al., 2011) can shadow bad research practices even when pre-registration works as intended. In doing so, we suggest the need for the evolution of the preregistration revolution (Nosek, Ebersole, DeHaven, \& Mellor, 2018). 
Table 1. Rerolling and analyzing performed in this study

\begin{tabular}{|c|c|c|c|c|c|c|c|}
\hline Data & Analysis & Results*1 & Significance & Hypothesizable? & Data processing & Reason for discarding & Report \\
\hline \multirow{9}{*}{1} & Binomial test & $p=0.4175$ & n.s. & & full data & & No \\
\hline & Fisher's exact test & $\underline{p}=0.5005$ & n.s. & & average split & & No \\
\hline & & $\underline{p}=0.5005$ & n.s. & & median split & & No \\
\hline & & $\underline{p}=0.2375$ & n.s. & & $10 \%$ top and bottom groups & & No \\
\hline & & $\underline{p}=0.2118$ & n.s. & & $15 \%$ top and bottom groups & & No \\
\hline & & $\underline{p}=0.2754$ & n.s. & & $20 \%$ top and bottom groups & & No \\
\hline & & $\underline{p}=0.1927$ & n.s. & & $25 \%$ top and bottom groups & & No \\
\hline & & $\underline{p}=0.2356$ & n.s. & & $30 \%$ top and bottom groups & & No \\
\hline & & $\underline{p}=0.0891$ & n.s. & & average $\pm 1 \mathrm{SD}$ groups & & No \\
\hline \multirow{10}{*}{2} & \multicolumn{7}{|c|}{ An ACQ was introduced } \\
\hline & Binomial test & $\underline{p}=0.6416$ & n.s. & & full data & & No \\
\hline & Fisher's exact test & $p=0.1432$ & n.s. & & average split & & No \\
\hline & & $\underline{p}=0.0832$ & n.s. & & median split & & No \\
\hline & & $\underline{p}=0.3852$ & n.s. & & $10 \%$ top and bottom groups & & No \\
\hline & & $\underline{p}=0.0951$ & n.s. & & $15 \%$ top and bottom groups & & No \\
\hline & & $\underline{p}=0.1591$ & n.s. & & $20 \%$ top and bottom groups & & No \\
\hline & & $p=0.0267$ & significant & Hard & $25 \%$ top and bottom groups & STAI-S high group $<$ STAI-S low group & No \\
\hline & & $\underline{p}=0.0677$ & significant & Hard & $30 \%$ top and bottom groups & STAI-S high group $<$ STAI-S low group & No \\
\hline & & $\underline{p}=0.2132$ & n.s. & & average $\pm 1 \mathrm{SD}$ groups & & No \\
\hline \multirow{10}{*}{3} & \multicolumn{7}{|c|}{ Two more ACQs were introduced } \\
\hline & Binomial test & $\underline{p}=0.9626$ & n.s. & & full data & & No \\
\hline & Fisher's exact test & $p=0.0920$ & n.s. & & average split & & No \\
\hline & & $p=0.0920$ & n.s. & & median split & & No \\
\hline & & $\underline{p}=0.0011$ & significant & Hard & $10 \%$ top and bottom groups & PANAS-Negative high group $<$ PANAS-Negative low group & No \\
\hline & & $\underline{p}=0.0006$ & significant & Hard & $15 \%$ top and bottom groups & PANAS-Negative high group $<$ PANAS-Negative low group & No \\
\hline & & $\underline{p}=0.0032$ & significant & Hard & $20 \%$ top and bottom groups & PANAS-Negative high group $<$ PANAS-Negative low group & No \\
\hline & & $p=0.0972$ & n.s. & & $25 \%$ top and bottom groups & & No \\
\hline & & $\underline{p}=0.1081$ & n.s. & & $30 \%$ top and bottom groups & & No \\
\hline & & $\underline{p}=0.0026$ & significant & Hard & average $\pm 1 \mathrm{SD}$ groups & PANAS-Negative high group $<$ PANAS-Negative low group & No \\
\hline \multirow{9}{*}{4} & Binomial test & $\underline{p}=0.3888$ & n.s. & & full data & & No \\
\hline & Fisher's exact test & $p=0.1019$ & n.s. & & average split & & No \\
\hline & & $\underline{p}=0.1731$ & n.s. & & median split & & No \\
\hline & & $p=0.1004$ & n.s. & & $10 \%$ top and bottom groups & & No \\
\hline & & $\underline{p}=0.0545$ & n.s. & & $15 \%$ top and bottom groups & & No \\
\hline & & $\underline{p}=0.0661$ & n.s. & & $20 \%$ top and bottom groups & & No \\
\hline & & $\underline{p}=0.0346$ & significant & Hard & $25 \%$ top and bottom groups & STAI-S high group $<$ STAI-S low group & No \\
\hline & & $p=0.0694$ & n.s. & & $30 \%$ top and bottom groups & & No \\
\hline & & $\underline{p}=0.0964$ & n.s. & & average $\pm 1 \mathrm{SD}$ groups & & No \\
\hline \multirow{9}{*}{5} & Binomial test & $\underline{p}=0.0049$ & significant & Easy & full data & hypothesizable but weak (left choice $>$ right choice) & No \\
\hline & Fisher's exact test & $\underline{p}=0.2323$ & n.s. & & average split & & No \\
\hline & & $\underline{p}=0.2323$ & n.s. & & median split & & No \\
\hline & & $p=0.6780$ & n.s. & & $10 \%$ top and bottom groups & & No \\
\hline & & $\underline{p}=0.3066$ & n.s. & & $15 \%$ top and bottom groups & & No \\
\hline & & $\underline{p}=0.5358$ & n.s. & & $20 \%$ top and bottom groups & & No \\
\hline & & $\underline{p}=0.2656$ & n.s. & & $25 \%$ top and bottom groups & & No \\
\hline & & $\underline{p}=0.1102$ & n.s. & & $30 \%$ top and bottom groups & & No \\
\hline & & $\underline{p}=0.3066$ & n.s. & & average $\pm 1 \mathrm{SD}$ groups & & No \\
\hline \multirow{10}{*}{6} & \multicolumn{7}{|c|}{ Positions of cards and buttons were changed } \\
\hline & Binomial test & $\underline{p}=0.0963$ & n.s. & & full data & & No \\
\hline & Fisher's exact test & $p=0.1904$ & n.s. & & average split & & No \\
\hline & & $\underline{p}=0.1904$ & n.s. & & median split & & No \\
\hline & & $\underline{p}=0.2908$ & n.s. & & $10 \%$ top and bottom groups & & No \\
\hline & & $\underline{p}=0.3716$ & n.s. & & $15 \%$ top and bottom groups & & No \\
\hline & & $\underline{p}=0.1628$ & n.s. & & $20 \%$ top and bottom groups & & No \\
\hline & & $\underline{p}=0.1636$ & n.s. & & $25 \%$ top and bottom groups & & No \\
\hline & & $\underline{p}=0.3149$ & n.s. & & $30 \%$ top and bottom groups & & No \\
\hline & & $\underline{p}=0.3038$ & n.s. & & average $\pm 1 \mathrm{SD}$ groups & & No \\
\hline
\end{tabular}




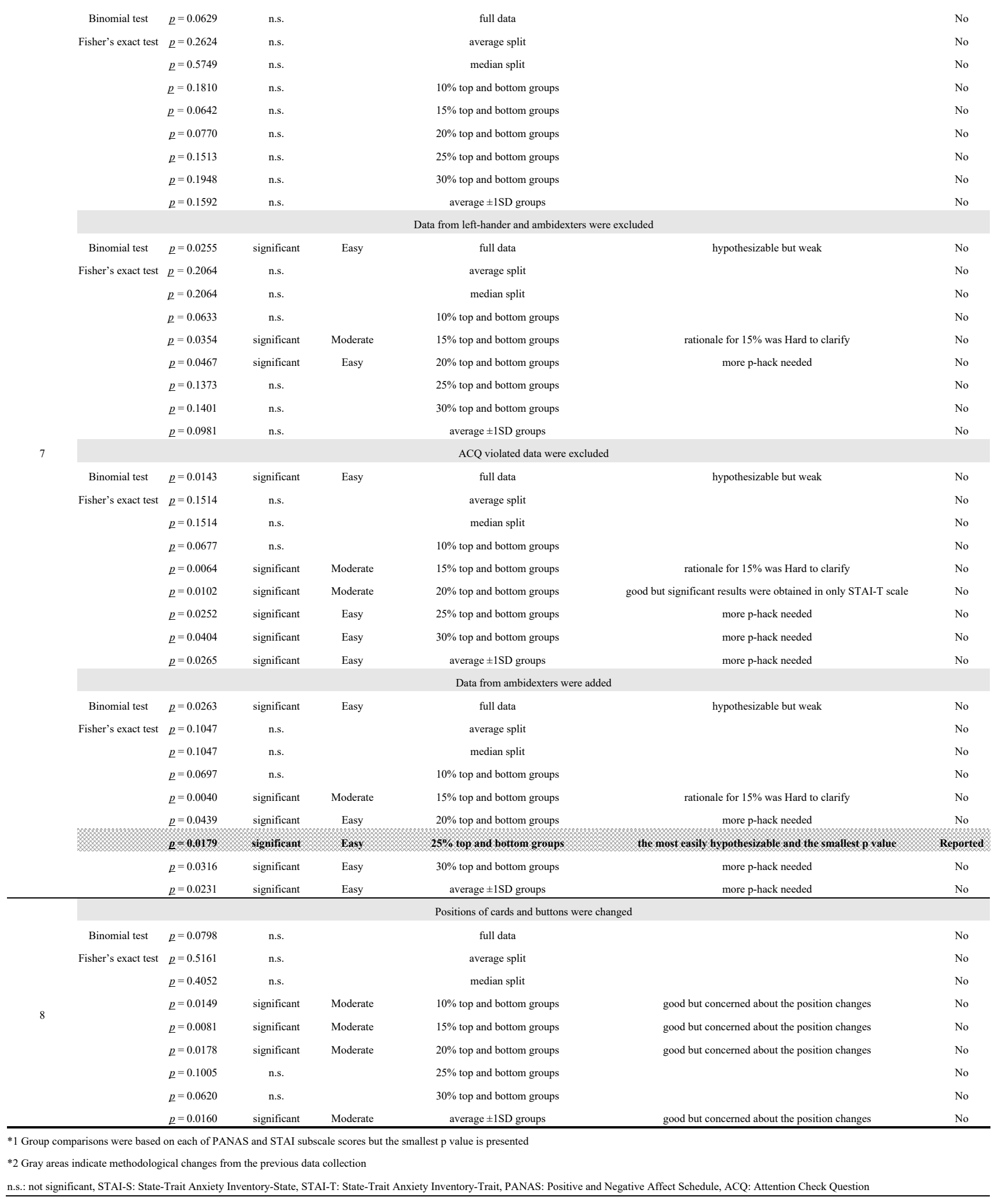


What types of QRPs were used in the present demonstration study? Detailed introductions of each QRP are as follows.

1. $\underline{P}$-hacking. First, to make the $\underline{p}$-value smaller, we increased the number of participants until we obtained statistically significant results. Second, we divided participants into two groups on an arbitrary basis (e.g., 10, 15, 20, 25, and 30\% top and bottom scores in a given scale) and repeated the analysis (99 times in total; see Table 1). Third, we arbitrarily used and managed ACQs and handedness as exclusion criteria to obtain the significant results we wanted.

2. HARKing. We created our working hypothesis that was presented in the introduction section after identifying results that were both significant with the smallest $\underline{p}$-value and had hypotheses that were easy to develop (i.e., hypothesizable; Table 1). Of course, we came up with a story and wrote the introduction after the results and the final hypotheses were determined. The power analysis was also carried out after the results were known.

3. Cherry picking. In every experiment, we obtained data using four psychological scales, STAI-S (State-Trait Anxiety Inventory-State; Spielberger, Gorsuch, \& Lushene, 1970), STAI-T (StateTrait Anxiety Inventory-Trait), PANAS-Positive, and PANAS-Negative. However, we only reported the scores of PANAS-Negative subscale because these data were the only set of significant results with an easily developed hypothesis. The data and results from the other scales were concealed from the method section as well as the results section.

4. Rerolling. We repeated the experiment eight times until desirable results were obtained (Table 1). In four of the eight experiments, we relocated the position of the response button rightward in the display directly before the third choice to attract a subsequent right-biased choice, and that indeed increased a general tendency of the right card choice (in the 5th-8th experiments). Only in the 6th and 8th experiments did we also change the positions of cards leftward to draw participants' attention to the right card that had been relocated toward the fovea. We reported the results that were from only one of these eight experiments (i.e., the 7th experiment) and elected not to mention 
the other experiments and what manipulations were deliberately done. In general, the method section does not provide complete information on all the stimuli, demonstrating how easily researchers can engage in these practices. The supplementary information necessary for data sharing contains only these partial data.

5. PARKing. We registered the hypothesis and the protocol after the results were known. All the shared data in the supplementary information were copied from a file and pasted to a new blank file after pre-registration. These data were obtained before pre-registration, and therefore, they were pasted to a new file later to ensure that the date information of the uploaded file appears to be after pre-registration.

6. Overissuing. We registered multiple similar protocols at numerous registration systems simultaneously. Specifically, at OSF (https://osf.io/4mde8/), AsPredicted (\#18254, https://aspredicted.org/mx7y9.pdf; \#22019, $\quad$ https://aspredicted.org/sv9nq.pdf), $\quad$ EGAP (\#20181230AA, http://egap.org/registration/5418), and AEA RCT Registry (where it was submitted but withdrawn because the study did not fall within the scope of RCT registries). In particular, in AsPredicted, two protocols with the opposite hypotheses were registered, but there is no way for anyone to know this other than the authors. The present study was conducted by performing PARKing, and therefore, this overissuing method is less useful, but we performed this QRP as a demonstration. If PARKing is not used, this method would be useful for rerolling. If researchers conduct several similar experiments at the same time and register them all in separate pre-registration systems, they can publish a "good" paper by reporting only the study with significant results. During peer review, no one can retrieve registered information on the other protocols from one specific system. For the editor and reviewer, the paper would appear to be excellent research with good results that were obtained following pre-registration.

7. Fake ethics statement. Although it is not a major point in the present study, we reported a fake approval number of ethical review (QU-07121981). The correct approval number was 2016-017 
$(07 / 12 / 1981$ is the birthday of one of the authors, YY). In the current ethical review, the content is rarely checked by third parties. It seems better to integrate the pre-registration and the ethical review process to ensure that ethical guidelines were followed.

8. Fictitious references. We cited some imaginary articles (i.e., Decks \& Pegg, 2001; Livisio, 1988; Saramista et al., 2015). Citations are often used to emphasize the importance of an author's claim, but only those who are fully versed in the field will recognize fake sources. Here, we used fictional author names to avoid any influence on actual researchers. A more practical way to do this practice is to mislead readers by intentionally citing false articles with the names of actual authors and by adding them in the references section. In this case, it is easy to say that it was just a mistake. Readers typically do not directly confirm each of the references in an article. This tendency becomes stronger when it is challenging to obtain journals or volumes because there are only available in print in certain locations or have been discontinued. In this way, malicious authors may attempt to increase the credibility of their findings by inserting false citations to provide credibility cue since the cited articles have been published in peer-reviewed journals.

The QRPs demonstrated in this article varied widely, from those directly related to the preregistration system to those not related at all. Figure 3 shows the relationship of the QRPs used here in terms of pre-registration. An important point demonstrated here is how to manipulate hypothesis testing by cracking the pre-registration system itself (e.g., PARKing and overissuing). As described later, these practices can be classified into those that can be easily patched and those that cannot be patched. Another important point is that there are some QRPs that cannot be prevented even if the preregistration system functions properly (e.g., rerolling). 
How can we prevent QRPs while preserving the pre-registration system? Indeed, the current pre-registration system can prevent many QRPs with a little ingenuity. For example, overissuing can be detected by developing a metasearch engine that covers multiple pre-registration systems. However, it is important for journal editors and reviewers to be able to search for the protocols even during the embargo period. If one is concerned that the blindedness of the submitted manuscript will be sacrificed, a dedicated reviewer who only checks for similarities between registered protocols and manuscripts could be used. Recently, the workload required of one reviewer has increased, which may contribute to an increase in the number of researchers refusing peer reviews. Reviewers' division of labor has been adopted by some journals (i.e., statistical reviewers: Petrovecki, 2009) that believe introducing "registration reviewers" can be crucial in reducing the traditional reviewer's efforts and increasing detectability of the sloppy use of the pre-registration system (e.g., Ioannidis, Caplan, \& Dal-Ré, 2017), as well as the pre-registration QRPs demonstrated here. Eventually, it will lead to the implementation of crowd peer-reviews as recently tested and used in Synlett, SynOpen, and Atmospheric Chemistry and Physics (List, 2017).

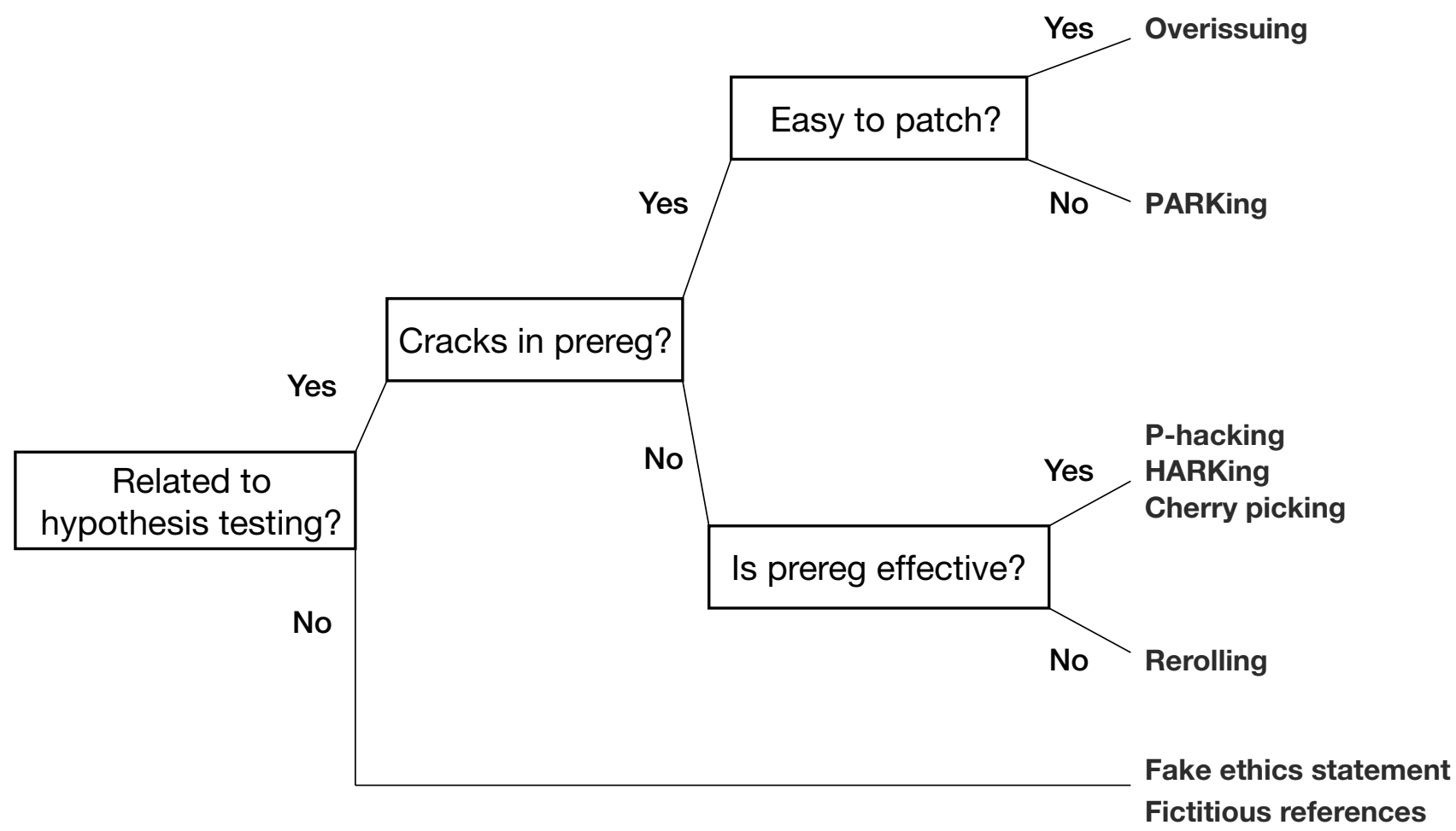

Figure 3. Classification of the QRPs used in this article. (Prereg means pre-registration.) 
In addition, rigorous peer-reviews in the first stage of pre-registration that call for changes to the experimental protocol would decrease the merits of PARKing in which experiments are conducted in advance. It should be noted that, in general, if there are unavoidable circumstances in which a preregistered protocol cannot be performed exactly, it can be accepted by making the protocol open. This leaves the possibility that after an initial peer-review, the protocol will be returned to the experimental method that was used before submission to pre-registration. In fact, a recent study showed that all preregistered published studies in Psychological Science deviated from the registered protocol (Claesen, Gomes, Tuerlinckx, \& Vanpaemel, 2019). Moreover, at this point, rerolling cannot be prevented by the pre-registration system, as there is no way to know how many times researchers conducted the same experiment before reporting it in the pre-registration system. To address this issue, it has been suggested that experiments should be video-recorded (Gilmore \& Adolph, 2017), and only the data obtained during the experiment can be accepted. Of course, video-recording can only guarantee the credibility of the data of the "finally submitted" experiment, and it cannot guarantee whether it is the best data obtained via rerolling. In this way, a cat-and-mouse game between system patchers and malicious researchers will continue.

For the pre-registration system to increase its credibility, greater reform is necessary. Yamada (2018) argued that outsourcing for experiments would be effective, that is, another researcher conducts the experiment with the pre-registered protocol, and as long as the researchers have no conflict of interest among them, there would be no need to engage in QRPs by the researcher who conducted experiments. Of course, how to bring together such experimenters is a problem (Romero, 2018). Now, this may be facilitated by implementing StudySwap, an online collaboration system (Chartier, Riegelman, \& McCarthy, 2018). Similarly, it would be effective to use crowdsourcing for the distributed experimentation (Uhlmann, Chartier, Ebersole, Errington, Kidwell, Lai et al., 2018). An advanced pre-registration system would be able to combine such methods to make experiments fair, unbiased, transparent, and highly reproducible. Instead of trusting a single experimental result by a 
single research team, triangulation of the experiment using different researchers and methodologies is needed (Gall, Ioannidis, \& Maniadis, 2017; Munafò, \& Smith, 2018). Implementing practical means to improve the replicability of experimental findings may slow the progress of research, but at this point, this is the only way to make psychology a field of science.

Readers may have different opinions about whether the practices demonstrated in this paper represent QRPs or scientific misconduct. For example, previous researchers who read an early draft of this paper indicated that PARKing constitutes outright fraud. The definition of scientific misconduct varies by field, country, organization, and researcher. Here, we treat FFP as scientific misconduct, according to the definition of the National Academy of Sciences, National Academy of Engineering, and Institute of Medicine (1992). On the other hand, in Nordic countries, scientific dishonesty is defined as a "violation against good scientific practice," "serious deviation from accepted ethical research practice," or "intentional distortion of the research process" (Nylenna, Andersen, Dahlquist, Sarvas, \& Aakvaag, 1999). As pre-registration has not yet become a normative research practice, it is unclear whether PARKing deviates from the ethical range. The pre-registration system allows researchers to report that their study is registered before any research activities. Therefore, PARKing will at least include false declarations. Then, how should we handle such false declarations? If treated as fraud, then by mandating the declaration of other QRPs at pre-registration, it may also be possible to treat them as fraud if it is found that they were implemented, and to impose a hard punishment. For example, if researchers are required to disclose all mutually similar registered protocols and future protocols, it would be fraud if they do not comply, which will prevent overissuing. We will discuss the boundary between QRPs and scientific misconduct in more detail in another article.

The present study, however, has a limitation. It is a hoax study, which may remind some readers of the Sokal affair (Sokal, 1996), Bohannon's case (Bohannon, 2013), or the case in which an IEEE conference accepted an automatically generated article (Schlangemann, 2008). These incidents have indicated the problems of academic publishing through the shocking method of publication of 
hoax papers. On the other hand, we did not submit our demonstration study itself to any journal. This is the limitation of the present research; the actual harmfulness of the QRPs used in the demonstration study remains unclear. The ethical concern of submitting a hoax paper to journals seemed to outweigh the benefits related to the impact of its successful acceptance and publication, and therefore, we decided not to submit it to any journal. However, this paper primarily aimed to make the existence of QRPs in pre-registration widely known, regardless of whether the demonstration study itself can be accepted. Thus, this paper is significant in that it conducted this demonstration, and this is not influenced by the above-mentioned limitation.

In summary, through this study, we aimed to inform readers of the existence of the new QRPs related only to pre-registration systems by demonstrating a study, and to share the sense of crisis that surrounds them. In the demonstration, we presented as much ordinary registered research as possible. We suggested that it was difficult to identify many QRPs from the face of the paper. In the general discussion, we reviewed all QRPs (e.g., p-hacking, HARKing, rerolling, and PARKing) that were performed in the pre-registered demonstration study. Finally, we claimed the necessity of further promoting transparency in our research practice. We suggested a division of labor, including crowdsourcing, in research and peer review. The aim is to decentralize costs and conflicts of interests of scientific players involved in research, cutting benefits to performing research misconduct and QRPs. Pre-registration is good but not perfect, which is quite natural, and therefore, to make it conscious and relevant, it was necessary to present specific examples. Although it is important to make research transparent, it is meaningless unless we correct it when we receive criticisms. Therefore, a more transparent and self-correcting research system is needed. 


\section{Footnotes}

1. This study does not exist. See "8. Fictitious references" in General Discussion. 


\section{Acknowledgments}

This research was supported by JSPS KAKENHI (15H05709, 16H01866, 17H00875, 18H04199, and $18 \mathrm{~K} 12015)$

\section{Author Contributions}

Contributed to conception and design: AI, XH, HN, ZS, YY

Contributed to acquisition of data: $\mathrm{AI}, \mathrm{XH}$

Contributed to analysis and interpretation of data: AI, XH, HN, ZS, YY

Drafted and/or revised the article: AI, XH, HN, ZS, YY

Approved the submitted version for publication: AI, XH, HN, ZS, YY

\section{Competing Interests}

The authors declare no competing financial interests.

\section{Data Availability}

The datasets are available at https://doi.org/10.6084/m9.figshare.7977035.v1 


\section{References}

Agnoli, F., Wicherts, J. M., Veldkamp, C. L. S., Albiero, P., \& Cubelli, R. (2017). Questionable research practices among italian research psychologists. $\underline{P L O S O N E}, \underline{12}(3), \mathrm{e} 0172792$.

Ayduk, O., Downey, G., Testa, A., Yen, Y., \& Shoda, Y. (1999). Does rejection elicit hostility in rejection sensitive women? Social Cognition, 17(2), 245-271.

Bem, D. J. (2004). Writing the empirical journal article. In Darley, J. M., Zanna, M. P., \& Roediger III, H. L. (Eds.), The compleat academic: A career guide (2nd ed., pp.185-219). Washington, DC: American Psychological Association.

Bem, D. J. (2011). Feeling the future: Experimental evidence for anomalous retroactive influences on cognition and affect. Journal of Personality and Social Psychology, 100(3), 407-425.

Bernard, C. (2017). Editorial: Code case - Investigating transparency and reproducibility. eNeuro, 4(4), e0233-17.

Boccia, S., Rothman, K. J., Panic, N., Flacco, M. E., Rosso, A., Pastorino, R., et al. (2016). Registration practices for observational studies on ClinicalTrials.gov indicated low adherence. Journal of Clinical Epidemiology, 70(C), 176-182.

Bohannon, J. (2013). Who's afraid of peer review? Science, 342(6154), 60-65.

Camerer, C. F., Dreber, A., Forsell, E., Ho, T. H., Huber, J., Johannesson, M., et al. (2016). Evaluating replicability of laboratory experiments in economics. Science, 351(6280), 1433-1436.

Casasanto, D. (2009). Embodiment of Abstract concept: Good and bad in right- and left-handers. Journal of Experimental Psychology: General, 138(3), 351-367.

Casasanto, D., \& Chrysikou, E.G., (2011). When left is "right": Motor fluency shapes abstract concept. Psychological Science, 22(4), 419-422.

Casasanto, D., \& Henetz, T. (2012). Handedness shapes children's abstract concepts. Cognitive Science, 36(2), 359-372. 
Casasanto, D., \& Jasmin, K. (2010). Good and bad in the hands of politicians: Spontaneous gestures during positive and negative speech. PLOS ONE, 5(7), e11805.

Chabris, C. F., Heck, P. R., Mandart, J., Benjamin, D. J., \& Simons, D. J. (2018). No evidence that experiencing physical warmth promotes interpersonal warmth: Two failures to replicate Williams and Bargh (2008). Social Psychology, 1-6.

Chandler, J., Mueller, P., \& Paolacci, G. (2014). Nonnaïveté among Amazon Mechanical Turk workers: Consequences and solutions for behavioral researchers. Behavior Research Methods, $\underline{46}(1), 112-130$.

Chartier, C. R., Riegelman, A., \& McCarthy, R. J. (2018). StudySwap: A platform for interlab replication, collaboration, and resource exchange. Advances in Methods and Practices in Psychological Science, 1 (4), 574-579.

Claesen, A., Gomes, S. L. B. T., Tuerlinckx, F., \& Vanpaemel, W. (2019). Preregistration: Comparing dream to reality. PsyArXiv, https://doi.org/10.31234/osf.io/d8wex

Colombo, M., Duev, G., Nuijten, M. B., \& Sprenger, J. (2018). Statistical reporting inconsistencies in experimental philosophy. PLoS ONE, $\underline{13}(4), \mathrm{e} 0194360$.

Corbetta, M., \& Shulman, G.L. (2002). Control of goal-directed and stimulus- driven attention in the brain. Nature Reviews Neuroscience, 3(3), 201-215.

Cova, F., Strickland, B., Abatista, A., Allard, A., Andow, J., Attie, M., et al. (2018). Estimating the Reproducibility of Experimental Philosophy. Review of Philosophy and Psychology, 28(11), $1-36$.

Erwin, B. A., Heimberg, R. G., Schneier, F. R., \& Liebowitz, M. R. (2003). Anger experience and expression in social anxiety disorder: Pretreatment profile and predictors of attrition and response to cognitive-behavioral treatment. Behavior Therapy, 34(3), 331-350.

Eysenck, M. W. (1982). Attention and arousal: Cognition and performance. Berlin: Springer-Verlag. 
Eysenck, M. W., Derakshan, N., Santos, R., \& Calvo, M. G. (2007). Anxiety and cognitive performance: attentional control theory. Emotion, $\underline{7}(2), 336$.

Faul, F., Erdfelder, E., Buchner, A., \& Lang, A. G. (2009). Statistical power analyses using G* Power 3.1: Tests for correlation and regression analyses. Behavior Research Methods, $\underline{41}$ (4), 11491160.

Fraser, H., Parker, T., Nakagawa, S., Barnett, A., \& Fidler, F. (2018). Questionable research practices in ecology and evolution. PLOS ONE 13(7), e0200303

Fujishima, Y., \& Higuchi, M. (2016). Case studies of "p-hacking” in social psychology. Japanese Psychological Review, $\underline{59}(1), 84-97$. (in Japanese)

Gall, T., Ioannidis, J. P. A., \& Maniadis, Z. (2017). The credibility crisis in research: Can economics tools help? PLoS Biology, 15(4), e2001846.

Galak, J., LeBoeuf, R. A., Nelson, L. D., \& Simmons, J. P. (2012). Correcting the past: Failures to replicate psi. Journal of Personality and Social Psychology, 103(6), 933-948.

Gilmore, R. O., \& Adolph, K. E. (2017). Video can make behavioural science more reproducible. Nature Human Behaviour, 1 (7), 0128.

Greenwald, A. G., Pratkanis, A. R., Leippe, M. R., \& Baumgardner, M. H. (1986). Under what conditions does theory obstruct research progress? Psychological Review, 93(2), 216-229.

Ioannidis, J. P. A. (2005). Contradicted and initially stronger effects in highly cited clinical research. $\underline{J A M A}, \underline{294}(2), 218-228$.

Ioannidis, J. P., Caplan, A. L., \& Dal-Ré, R. (2017). Outcome reporting bias in clinical trials: Why monitoring matters. $\underline{B M J}, \underline{356}, \mathrm{j} 408$.

John, L. K., Loewenstein, G., \& Prelec, D. (2012). Measuring the prevalence of questionable research practices with incentives for truth telling. Psychological Science, 23(5), 524-532.

Jonas, K. J., \& Cesario, J. (2016). How can preregistration contribute to research in our field? Comprehensive Results in Social Psychology, 1(1-3), 1-7. 
Kashdan, T. B., Collins, R. L., \& Elhai, J. D. (2006). Social anxiety and positive outcome expectancies on risk-taking behaviors. Cognitive Therapy and Research, $\underline{30}(6), 749-761$.

Kawahito, J., Otsuka, Y., Kaida, K., \& Nakata, A. (2011). Reliability and validity of the Japanese version of 20-item Positive and Negative Affect Schedule. Hiroshima Psychological Research, 11, 225-240.

Kerr, N. L. (1998). HARKing: Hypothesizing after the results are known. Personality and Social Psychology Review, 2(3), 196-217.

Klein, R. A., Vianello, M., Hasselman, F., Adams, B. G., Adams, R. B., Jr., Alper, S., et al. (2018). Many labs 2: Investigating variation in replicability across samples and settings. $\underline{\text { Advances in }}$ Methods and Practices in Psychological Science, 1(4), 443-490.

Lösel, F. (2017). Evidence comes by replication, but needs differentiation: The reproducibility issue in science and its relevance for criminology. Journal of Experimental Criminology, 28(11), $1-22$.

List, B. (2017). Crowd-based peer review can be good and fast. Nature, $\underline{546}, 9$.

Lynott, D., Corker, K. S., Wortman, J., Connell, L., Donnellan, M. B., Lucas, R. E., \& O’Brien, K. (2014). Replication of “experiencing physical warmth promotes interpersonal warmth" by Williams and Bargh (2008). Social Psychology, 45(3), 216-222.

Mahoney, M. J. (1977). Publication prejudices: An experimental study of confirmatory bias in the peer review system. Cognitive Therapy and Research, 1(2), 161-175.

Marmolejo-Ramos, F., Elosúa, M. R., Yamada, Y., Hamm, N. F., \& Noguchi, K. (2013). Appraisal of space words and allocation of emotion words in bodily space. $\underline{P L O S O N E}, \underline{8}(12)$, e81688.

Mellor, D. T., \& Nosek, B. A. (2018). Easy preregistration will benefit any research. Nature Human Behaviour, 2(2), 98 . 
Motyl, M., Demos, A. P., Carsel, T. S., Hanson, B. E., Melton, Z. J., Mueller, A. B., et al. (2017). The state of social and personality science: Rotten to the core, not so bad, getting better, or getting worse? Journal of Personality and Social Psychology, 113(1), 34-58.

Munafò, M. R., Nosek, B. A., Bishop, D. V. M., Button, K. S., Chambers, C. D., Sert, du, N. P., et al. (2017). A manifesto for reproducible science. Nature Human Behaviour, 1(1), 1-9.

Munafò, M. R., \& Smith, G. D. (2018). Repeating experiments is not enough. Nature, 553, 399-401.

National Academy of Sciences, National Academy of Engineering and Institute of Medicine. Panel on Scientific Responsibility and the Conduct of Research. (1992). Responsible science, Volume I: Ensuring the integrity of the research process. Washington DC: National Academy Press.

Nosek, B. A., Ebersole, C. R., DeHaven, A. C., \& Mellor, D. T. (2018). The preregistration revolution. Proceedings of the National Academy of Sciences of the United States of America, 18 , 201708274.

Nosek, B. A., Spies, J. R., \& Motyl, M. (2012). Scientific Utopia: II. Restructuring incentives and practices to promote truth over publishability. Perspectives on Psychological Science, $\underline{7}(6)$, 615-631.

Nylenna, M., Andersen, D., Dahlquist, G., Sarvas, M., \& Aakvaag, A. (1999). Handling of scientific dishonesty in the Nordic countries. Lancet, 354(9172), 57-61.

Open Science Collaboration. (2015). Estimating the reproducibility of psychological science. Science, $\underline{349}(6251)$, aac4716.

Oppenheimer, D. M., Meyvis, T., \& Davidenko, N. (2009). Instructional manipulation checks: Detecting satisficing to increase statistical power. Journal of Experimental Social Psychology, $\underline{45}(4), 867-872$.

Petrovecki, M. (2009). The role of statistical reviewer in biomedical scientific journal. Biochemia Medica, $\underline{19}(3), 223-230$. 
Posner, M. I., \& Petersen, S. E. (1990). The attention system of the human brain. Annual Review of Neuroscience, $\underline{13}(1), 25-42$.

$\mathrm{R}$ Core Team (2017). $\underline{R}$ : A language and environment for statistical computing. $\mathrm{R}$ Foundation for Statistical Computing, Vienna, Austria. https://www.r-project.org/.

Ritchie, S. J., Wiseman, R., \& French, C. C. (2012). Failing the future: Three unsuccessful attempts to replicate Bem's 'retroactive facilitation of recall' effect. PLOS ONE, 7(3), e33423.

Romero, F. (2018). Who should do replication labor? Advances in Methods and Practices in Psychological Science, $\underline{1}(4), 516-537$.

Sasaki, K., Yonemitsu, F., \& Yamada, Y. (in press). Goodness of the side of the dominant hand: A registered direct replication of Casasanto (2009). Japanese Psychological Review.

Sasaki, K., \& Yamada, Y. (in press). Crowdsourcing visual perception experiments: A case of contrast threshold. PeerJ.

Schlangemann, H. (2008). Towards the simulation of e-commerce. Proceedings of the 2008 International Conference on Computer Science and Software Engineering, $\underline{5}, 1144-1147$.

Shaffer, D., Gould, M. S., Fisher, P., Trautman, P., Moreau, D., Kleinman, M., \& Flory, M. (1996). Psychiatric diagnosis in child and adolescent suicide. Archives of General Psychiatry, $\underline{53}(4)$, $339-348$

Simmons, J. P., Nelson, L. D., \& Simonsohn, U. (2011). False-positive psychology: Undisclosed flexibility in data collection and analysis allows presenting anything as significant. Psychological Science, 22(11), 1359-1366.

Sokal, A. (1996) Transgressing the boundaries. Towards a transformative hermeneutics of quantum gravity, Social Text, $\underline{46 / 47}, 217-252$.

Staal, M. A. (2004). Stress, cognition, and human performance: A literature review and conceptual framework. National Aeronautics and Space Administration (NASA) Technical Memorandum, TM-2004-212824. Moffett Field, CA: Ames Research Center. 
Sterling, T. D., Rosenbaum, W. L., \& Weinkam, J. J. (1995). Publication decisions revisited: The effect of the outcome of statistical tests on the decision to publish and vice versa. The American Statistician, 49(1), 108-112.

Strube, M. J. (2006). SNOOP: A program for demonstrating the consequences of premature and repeated null hypothesis testing. Behavior Research Method, 38(1), pp. 24-27

Spielberger, C. D., Gorsuch, R. L., \& Lushene, R. E. (1970). STAI: Manual for the State-Trait Anxiety Inventory. CA: Counseling Psychologist Press.

Szücs, D., \& Ioannidis, J. P. A. (2017). Empirical assessment of published effect sizes and power in the recent cognitive neuroscience and psychology literature. $\underline{P L O S \text { Biology, } 15}$ (3), e2000797.

Uhlmann, E. L., Chartier, C. R., Ebersole, C. R., Errington, T. M., Kidwell, M., Lai, C. K., ... Nosek, B. A. (2018, August 13). Scientific utopia III: Crowdsourcing science. PsyArXiv. https://doi.org/10.31234/osf.io/vg649

van 't Veer, A. E., \& Giner-Sorolla, R. (2016). Pre-registration in social psychology-A discussion and suggested template. Journal of Experimental Social Psychology, 67(C), 2-12.

Watson, D., Clark, L. A., \& Tellegen, A. (1988). Development and validation of brief measures of positive and negative affect: the PANAS scales. Journal of Personality and Social Psychology, 54(6), 1063-1070.

Williams, L. E., \& Bargh, J. A. (2008). Experiencing physical warmth promotes interpersonal warmth. Science, $\underline{322(5901), 606-607 .}$

Yamada, Y. (2018). How to crack pre-registration: Toward transparent and open science. Frontiers in Psychology, 9:1831. 